\title{
Preoperative Neutrophil-lymphocyte Ratio Is a Predictor of High-output Ileostomy After Colorectal Surgery
}

\author{
YUKI HIRAMOTO $^{1}$, HIDEJIRO KAWAHARA ${ }^{1}$, TOMO MATSUMOTO $^{1}$, \\ MITSUMASA TAKEDA ${ }^{1}$, TAKEYUKI MISAWA ${ }^{1}$ and KATSUHIKO YANAGA ${ }^{2}$ \\ ${ }^{1}$ Department of Surgery, Kashiwa Hospital, Jikei University School of Medicine, Chiba, Japan; \\ ${ }^{2}$ Department of Surgery, Jikei University School of Medicine, Tokyo, Japan
}

\begin{abstract}
Background/Aim: High-output ileostomy (HOI) can affect quality of life, however, its primary cause remains unknown. This study aimed to identify a predictor of $\mathrm{HOI}$ after colorectal surgery. Patients and Methods: The medical records of forty-five patients who had undergone colorectal surgery with temporary ileostomy without postoperative complications, such as intra-abdominal abscess, paralytic ileus, outlet obstruction, or suture rupture, at our hospital between January 2016 and December 2017 were retrospectively investigated. Results: Significant differences in age, gender, operative situation, duration of operation, intraoperative blood loss, operation procedure, operation approach, preoperative body mass index, and preoperative hematological/biochemical parameters, such as leucocyte counts, hemoglobin, serum total protein, albumin, C-reactive protein, and preoperative complications, were not identified between the two groups. Preoperative neutrophil-tolymphocyte ratio (NLR) of the HOI group was significantly higher than that of the non-HOI group $(p=0.004)$. Conclusion: Preoperative NLR seems to be a useful predictor of HOI after colorectal surgery.
\end{abstract}

High-output ileostomy (HOI) is often observed after anuspreserving procedures such as low anterior resection and intersphincteric resection, since an ileostomy is required to reduce the risk of severe anastomotic leakage (1-3). HOI occurs secondly to the intra-abdominal abscess and other similar conditions due to the resulting paralytic ileus that prevents absorption of small intestinal drainage (4-8) However, the primary cause of development of HOI remains

Correspondence: Yuki Hiramoto, MD. Department of Surgery, Kashiwa Hospital, Jikei University School of Medicine, 163-1 Kashiwashita, Kashiwashi, Chiba 277-8567, Japan. Tel: +81 471641111 Ext. 3421, Fax: +81 471633488, e-mail: yukihiramoto06190625@gmail.com

Key Words: High-output ileostomy, neutrophil-to-lymphocyte ratio, colorectal surgery. unknown. This study aimed to identify a predictor of HOI after colorectal surgery.

\section{Patients and Methods}

Patients. The Ethics Committee for Biomedical Research of the Jikei Institutional Review Board approved the protocol [30415(9436)], and all patients or their family members provided their written informed consent to participation. Between January 2016 and December 2017, forty-five patients who had undergone colorectal surgery with temporary ileostomy without postoperative complications, such as intra-abdominal abscess, paralytic ileus, outlet obstruction, or suture rupture, at our hospital were identified and their medical records were retrospectively examined. Ileostomy was created approximately $30-40 \mathrm{~cm}$ away from the ileocecal valve or iliac pouch in all patients, who had the same postoperative care pathway. HOI was defined when the maximum output was more than $2,000 \mathrm{ml} /$ day for three or more consecutive days and biochemical disturbances were imminent (7).

This study included 22 patients with high-output ileostomy (HOI) and 23 patients with non-high-output ileostomy (non-HOI). The hematological/biochemical parameters measured before surgery included levels of leucocyte counts, hemoglobin, serum total protein, albumin, and C-reactive protein. Preoperative neutrophil to lymphocyte ratio (NLR) was calculated by the number of neutrophils and lymphocytes. Body mass index (BMI) was calculated using the standard formula: weight $(\mathrm{kg}) /$ height $\left(\mathrm{m}^{2}\right)$.

Statistical analysis. Continuous variables are expressed as the means and ranges. The Wilcoxon rank-sum test was used to compare the continuous variables, and the Chi-square test was used to compare the categorical data. The receiver operating characteristic (ROC) curve analysis was used to determine an optimal cut-off value categorized for the two groups (9). A $p$-value of less than 0.05 was used to indicate significance. All data were analyzed using the Statistical Package for Social Sciences (SPSS) 24.0, (IBM SPSS, Tokyo, Japan).

\section{Results}

Comparison of clinical features between the non-HOI and the HOI group. The mean age of the HOI group was 56.0 years (range $=20-77$ years), while that of the non-HOI group was 63.0 years (range $=28-85$ years). There was not significant 
Table I. Comparison of clinical features between the HOI and the non-HOI group.

\begin{tabular}{|c|c|c|c|}
\hline Characteristic & HOI group $(n=22)$ & Non-HOI group $(n=23)$ & $p$-Value \\
\hline Mean age (range), years & $56.0(20-77)$ & $63.0(28-85)$ & 0.186 \\
\hline Gender, $\mathrm{n}(\%)$ & & & 0.873 \\
\hline Male & $10(45)$ & $11(48)$ & \\
\hline Female & $12(55)$ & $12(52)$ & \\
\hline Postoperative hospital stay (rang), days & $38.8(18-90)$ & $15.3(12-22)$ & $<0.001$ \\
\hline Emergency operation & & & 0.912 \\
\hline Presence, n (\%) & $8(36)$ & $8(35)$ & \\
\hline Absence, n (\%) & $14(64)$ & $15(65)$ & \\
\hline Operative time (range), min & $217.2(133-374)$ & $211.5(105-329)$ & 0.871 \\
\hline Blood loss (range), $\mathrm{ml}$ & $86.4(0-490)$ & $73.0(0-410)$ & 0.748 \\
\hline Operative procedure & & & 0.912 \\
\hline Laparosocpic surgery, n (\%) & $14(64)$ & $15(65)$ & \\
\hline Open surgery, $\mathrm{n}(\%)$ & $8(36)$ & $8(35)$ & \\
\hline Operative approach & & & 0.987 \\
\hline Proctocolectomy, n (\%) & $4(18)$ & $4(17)$ & \\
\hline Low anterior resection, $\mathrm{n}(\%)$ & $10(46)$ & $11(48)$ & \\
\hline Partial colectomy, n (\%) & $8(36)$ & $8(35)$ & \\
\hline Body mass index (range), $\mathrm{kg} / \mathrm{m}^{2}$ & $20.3(15.6-26.6)$ & $21.9(16.3-29.1)$ & 0.058 \\
\hline Leucocyte counts (range), $/ \mathrm{ml}$ & $7868.2(2300-14200)$ & $8017.4(3200-13800)$ & 0.786 \\
\hline NLR (range) & $7.3(3.1-34.5)$ & $2.5(1-3.4)$ & 0.004 \\
\hline Total protein (range), g/dl & $6.1(4.3-7.7)$ & $6.3(4.2-8.2)$ & 0.528 \\
\hline Albumin (range), $\mathrm{g} / \mathrm{dl}$ & $3.1(1.5-4.4)$ & $3.2(2-4.5)$ & 0.618 \\
\hline Hemoglobin (range), g/dl & $11.9(8.2-16.7)$ & $12.0(7.5-16)$ & 0.762 \\
\hline C-reactive protein (range), $\mathrm{g} / \mathrm{ml}$ & $3.8(0.1-20.4)$ & $3.0(0.1-11.5)$ & 0.546 \\
\hline Preoperative complications & & & 0.629 \\
\hline Heart disease, n $(\%)$ & $2(9)$ & $2(9)$ & \\
\hline Kidney disease, n (\%) & $0(0)$ & $0(0)$ & \\
\hline Diabetes mellitus, n (\%) & $2(9)$ & $1(4)$ & \\
\hline
\end{tabular}

HOI: High-output ileostomy; NLR: neutrophil/lymphocyte ratio.

difference between the two groups. Postoperative hospital stay of the HOI group was significantly longer than that of the nonHOI group. Significant differences in gender, operative situation, operation duration, intraoperative blood loss, operation procedure, operation approach, preoperative body mass index, and preoperative hematological/biochemical parameters such as leucocyte counts, hemoglobin, serum total protein, albumin, C-reactive protein, and preoperative complications, were not identified between the two groups. Whereas preoperative NLR of the HOI group was significantly higher than that of the non-HOI group $(p=0.004)$ (Table I).

The cut-off value of NLR categorized for the two groups. For categorizing for the two groups, the optimal cut-off value was calculated 3.0 by the ROC curve analysis, and the area under the curve (AUC) was 0.972 (Figure 1).

\section{Discussion}

Several studies have reported that HOI develops in $16-24 \%$ of patients after creation of ileostomy $(4,8,10)$. HOI can affect quality of life, however, its cause remains unknown. Factors such as intra-abdominal infections, temporary bowel obstruction, residual small bowel less than $200 \mathrm{~cm}$, enteritis such as Clostridium difficile (11) or salmonella, sudden drug withdraw (steroids, opiates), and the administration of prokinetic drugs such as metoclopramide (8) are considered to contribute to HOI development. Those secondary causes of development of HOI have been clearly identified, however, the primary cause of development of HOI after creation of ileostomy remains unknown. Therefore, the medical records of patients who had undergone colorectal surgery with temporary ileostomy without postoperative complications, such as intraabdominal abscess, paralytic ileus, outlet obstruction, or suture rupture, at our hospital were retrospectively examined.

Takeda has reported that diabetes and proctocolectomy were preoperative predictors of HOI after creation of ileostomy (10). In our study, preoperative NLR was the only identified potential preoperative predictor of HOI after colorectal surgery. The total leukocyte and neutrophil counts have historically been used as markers of infection. The NLR which is defined by the absolute number of neutrophils divided by the absolute number of lymphocytes is also considered an inflammatory biomarker. Several studies have 


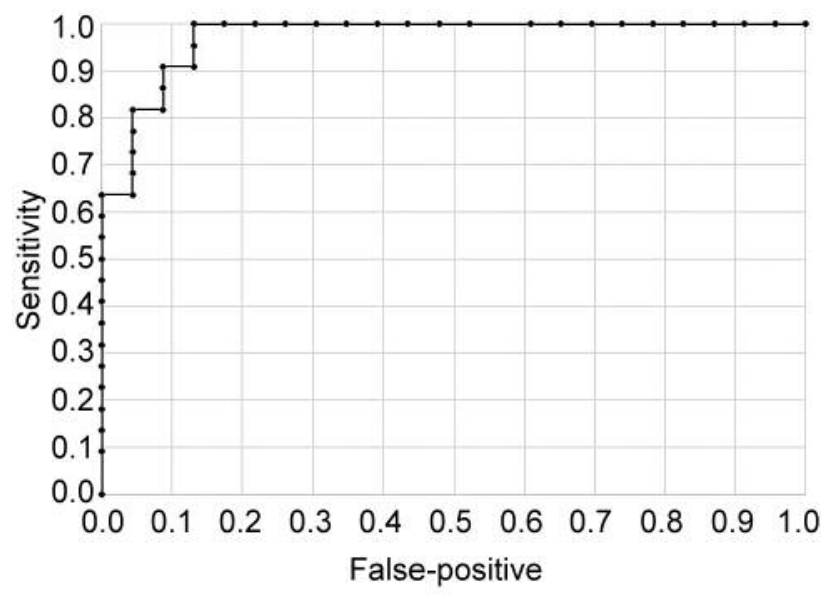

Figure 1. The receiver operating characteristic (ROC) curve analysis was used to determine an optimal cut-off value categorized for the two groups. The optimal cut-off value was calculated 3.0 by the ROC curve analysis and the area under the curve (AUC) was 0.972.

reported that an elevated NLR is associated with a poor prognosis in patients with various types of malignant diseases (12-17). However, the ideal cut-off value has still been controversial, however, there have been some reports that a cut-off value of more than 3-5 could be a useful predictor of the prognosis of patients with malignant diseases $(18,19)$.

In this present study, the optimal cut-off value categorized for the HOI group or the non-HOI group was 3.0 calculated by the ROC curve analysis. Because the NLR of the HOI group was significantly higher than that of the non-HOI group, immune depression may be strongly associated with HOI after creation of ileostomy. However, a large-scale prospective or retrospective study is needed to clarify this issue.

In conclusion, preoperative NLR seems to be a useful predictor of HOI after colorectal surgery.

\section{Conflicts of Interest}

The Authors declare that there are no conflicts of interest regarding this study.

\section{Authors' Contributions}

All Authors performed operations, analyzed the data of patients regarding their clinical features, and have been involved in drafting the manuscript. KY had given final approval of the version to be published. All Authors read and approved the final manuscript.

\section{References}

1 Matthiessen P, Hallböök O, Rutegård J, Simert G and Sjödahl $\mathrm{R}$ : Defunctioning stoma reduces symptomatic anastomotic leakage after low anterior resection of the rectum for cancer: A randomized multicenter trial. Ann Surg 246: 207-214, 2007. PMID: 17667498. DOI: 10.1097/SLA.0b013e3180603024

2 Veenhof AA, van der Peet DL, Meijerink WJ and Cuesta MA: Defunctioning stoma reduces symptomatic anastomotic leakage after low anterior resection of the rectum for cancer: A randomized multicenter trial. Ann Surg 247: 718-720, 2008. PMID: 18362645. DOI: 10.1097/ SLA.0b013e31816a7493

3 Katsuno H, Shiomi A, Ito M, Koide Y, Maeda K, Yatsuoka T, Hase K, Komori K, Minami K, Sakamoto K, Saida Y and Saito $\mathrm{N}$ : Comparison of symptomatic anastomotic leakage following laparoscopic and open low anterior resection for rectal cancer: A propensity score matching analysis of 1014 consecutive patients. Surg Endosc 30: 2848-2856, 2016. PMID: 26487228. DOI: $10.1007 / \mathrm{s} 00464-015-4566-2$

4 Nightingale J and Woodward JM: Guidelines for management of patients with a short bowel. Gut 55: 1-12, 2006. PMID: 16837533. DOI: $10.1136 /$ gut.2006.091108

5 Robertson I, Leung E, Hughes D, Spiers M, Donnelly L, Mackenzie I and Macdonald A: Prospective analysis of stomarelated complications. Colorectal Dis 7: 279-285, 2005. PMID: 15859968. DOI: 10.1111/j.1463-1318.2005.00785.x

6 Caricato M, Ausania F, Ripetti V, Bartolozzi F, Campoli G and Coppola R: Retrospective analysis of long-term defunctioning stoma complications after colorectal surgery. Colorectal Dis 9: 559-561, 2007. PMID: 17573753. DOI: 10.1111/j.1463-1318. 2006.01187.x

7 Cottam J, Richards K, Hasted A and Blackman A: Results of a nationwide prospective audit of stoma complications within 3 weeks of surgery. Colorectal Dis 9: 834-838, 2007. PMID: 17672873. DOI: 10.1111/j.1463-1318.2007.01213.x

8 Baker ML, Williams RN and Nightingale JM: Causes and management of a high-output stoma. Colorectal Dis 13: 191-197, 2011. PMID: 19888956. DOI: 10.1111/j.1463-1318. 2009. 02107.x

9 Hajian-Tilaki K: Receiver Operating Characteristic (ROC) Curve Analysis for Medical Diagnostic Test Evaluation. Caspian J Intern Med 4: 627-635, 2013. PMID: 24009950.

10 Takeda M, Takahashi H, Haraguchi N, Miyoshi N, Hata T, Yamamoto H, Matsuda C, Mizushima T, Doki Y and Mori M: Factors predictive of high-output ileostomy: a retrospective single-center comparative study. Surg Today, 2018. PMID: 30594951. DOI: 10.1007/s00595-018-1756-2

11 Williams RN, Hemingway D and Miller AS: Enteral Clostridium difficile, an emerging cause for high output ileostomy. J Clin Pathol 62: 951-953, 2009. PMID: 19447832. DOI: 10.1136/ jcp.2008.062901

12 Shimada H, Takiguchi N, Kainuma O, Soda H, Ikeda A, Cho A, Miyazaki A, Gunji H, Yamamoto $\mathrm{H}$ and Nagata M: High preoperative neutrophil-lymphocyte ratio predicts poor survival in patients with gastric cancer. Gastric Cancer 13: 170-176, 2010. PMID: 20820986. DOI: 10.1007/s10120-010-0554-3

13 Chua W, Charles KA, Baracos VE and Clarke SJ: Neutrophil/lymphocyte ratio predicts chemotherapy outcomes in patients with advanced colorectal cancer. Br J Cancer 104: 12881295, 2011. PMID: 21448173. DOI: 10.1038/bjc.2011.100

14 Wang GY, Yang Y, Li H, Zhang J, Jiang N, Li MR, Zhu HB, Zhang Q and Chen GH: A scoring model based on neutrophil to lymphocyte ratio predicts recurrence of $\mathrm{HBV}$-associated hepatocellular carcinoma after liver transplantation. PLoS One 6: e25295, 2011. PMID: 21966488. DOI: 10.1371/journal. pone. 0025295 
15 Motomura T, Shirabe K, Mano Y, Muto J, Toshima T, Umemoto Y, Fukuhara T, Uchiyama H, Ikegami T, Yoshizumi T, Soejima $Y$ and Maehara Y: Neutrophil-lymphocyte ratio reflects hepatocellular carcinoma recurrence after liver transplantation via inflammatory microenvironment. J Hepatol 58: 58-64, 2013. PMID: 22925812. DOI: 10.1016/j.jhep. 2012.08.017

16 Yoshizumi T, Ikegami T, Yoshiya S, Motomura T, Mano Y, Muto J, Ikeda T, Soejima Y, Shirabe K and Maehara Y: Impact of tumor size, number of tumors and neutrophil-to-lymphocyte ratio in liver transplantation for recurrent hepatocellular carcinoma. Hepatol Res 43: 709-716, 2013. PMID: 23190306. DOI: $10.1111 /$ hepr.12016

17 Gürol G, Çiftci İH, Terizi HA, Atasoy AR, Ozbek A and Köroğlu M: Are there standardized cut-off values for neutrophillymphocyte ratios in bacteremia or sepsis? J Microbiol Biotechnol 25: 521-525, 2015. PMID: 25341467.

18 Xiao GQ, Liu C, Liu DL, Yang JY and Yan LN: Neutrophillymphocyte ratio predicts the prognosis of patients with hepatocellular carcinoma after liver transplantation. World J Gastroenterol 19: 8398-8407, 2013. PMID: 24363533. DOI: $10.3748 /$ wjg. v19.i45.8398
19 Takakura K, Ito Z, Suka M, Kanai T, Matsumoto Y, Odahara S, Matsudaira H, Haruki K, Fujiwara Y, Saito R, Gocho T, Nakashiro K, Hamakawa H, Okamoto M, Kajihara M, Misawa $\mathrm{T}$, Ohkusa $\mathrm{T}$ and Koido S: Comprehensive assessment of the prognosis of pancreatic cancer: peripheral blood neutrophillymphocyte ratio and immunohistochemical analyses of the tumour site. Scand J Gastroenterol 51: 610-617, 2016. PMID: 26679084. DOI: $10.3109 / 00365521.2015 .1121515$

Received April 12, 2019

Revised April 30, 2019

Accepted May 2, 2019 